\title{
2
}

\section{Lists in Flux, Lives on Hold? Technologies of Waiting in Liver Transplant Medicine}

\author{
Julia Rehsmann
}

\section{Introduction: On Top of a Mountain}

Getting comfortable in his armchair, Arthur took another quick glance at the mobile phone on the coffee table next to him. While I was familiar with this glance from other social contexts-just quickly checking the phone before, or even during, a conversation-this look of his carried an existential urgency and uncertain expectation that shed light on the particularities of liver transplants. 'It wasn't that long ago', Arthur sighed, 'I had this terrible nightmare; I was on top of a mountain, enjoying the fresh air and the amazing view when my phone suddenly rang'. It was his transplant clinic calling him, telling him that he had to come in immediately because they finally had a donor liver for him. 'But standing on the mountain top, it was impossible for me to make it in time to the

J. Rehsmann ( $\otimes)$

Institute of Social Anthropology, University of Bern, Bern, Switzerland

Department of Health Professions, Bern University of Applied Science, Bern, Switzerland 
hospital', Arthur said and shook his head in disbelief. It was there on this mountain top that he missed his chance for a transplant.

In this chapter, I want to use Arthur's relation to his mobile phone in his dreams and his everyday life to unpack the ambivalent relationship between mobility and immobility when waiting for a transplant. Based on ethnographic research in Germany, I focus on the time before transplantation to discuss the role technologies play in the field of liver transplants-a field that is characterized by uncertainties, urgency and delay. In this particular temporality of transplant medicine, such technologies as mobile phones become a critical feature that shapes the waiting experiences of patients like Arthur Berger ${ }^{1}$ by mediating hope for a life-saving donor liver. Understanding waiting as a temporal experience that is shaped by hope, uncertainties and expectations, I scrutinize how the high-tech medical treatment of liver transplants works as a 'hope-generating machine' (Nuijten 2003, 16).

In what follows, I take a closer look at the crucial technological features of this hope-generating machine: how they are constituted, how they relate to each other, and how they affect patients. Inspired by Geoffrey C. Bowker and Susan Leigh Star's (2000) work on information infrastructures and classificatory practices, I discuss the waiting list as an invisible infrastructure and classifying technology of this hope-generating machine. Addressing the issue of visibility brings the materiality of lists to mind, and I delineate how waiting lists for transplants differ from common understandings of lists as a fixed, stable order, documented on paper. Furthermore, I show how these specially configured lists are based on the invisible workings of a complex computer algorithm. Handing decisions over to allocation algorithms makes it difficult to understand the workings of the waiting list and its conclusions. I contend that by being passed on to a computer program, decisions that have life and death consequences remain opaque for those affected by algorithms' calculations and assessments.

After discussing the particularities of lists in flux and the allocating algorithms they are based upon, I contend that the mobile phone becomes a critical feature in this invisible infrastructure. I argue that

${ }^{1}$ All names have been anonymized by the author. 
in the context of liver transplants, the mobile phone is more than a mere communication device: it becomes an extension of the invisible and intangible waiting list. Discussing Arthur's experience of waiting for a transplant, I demonstrate how, during waiting, the mobile phone becomes a tangible manifestation of the ephemeral waiting list, a mediator of hope for a life-saving treatment, as well as a reminder of one's dependency on medical care. I show how the mobile phone transforms in this context from a mere technological communication tool used to reach people at any time and which increases mobility for those carrying it into an ambivalent marker of people's simultaneous mobility and immobility.

\section{Methods}

This chapter is informed by 13 months of in-depth ethnographic liver transplant medicine in Germany that was conducted in 2014 and 2015 and supplemented by several short field trips in 2015-2017. I explored liver transplant medicine in a variety of locations, from transplant clinics to people's home, medical conferences to patient support group meetings. This allowed me to include a wide range of perspectives, from nurses, hepatologists and transplant surgeons, through administrative personnel and journalists to patients and their relatives, while focusing particularly on patients' experiences before as well as after transplant. Ethics approval was awarded by the University of Leipzig. Informed by multiple problem-centred, narrative interviews with 29 patients and 13 medical professionals and other experts, in this chapter, I present one exemplary patient case to discuss in detail how waiting for a liver transplant is bound up with very intimate considerations concerning life and death that are contingent on such technologies as algorithms, lists and mobile phones. 


\section{Transplant Medicine as a 'Hope-Generating Machine'}

With his liver failing due to cancer and cirrhosis, Arthur's fate was bound up with the medical possibilities transplant medicine in Germany had to offer. As his failing and tumorous liver would inevitably lead to his death, Arthur had placed all his hope on a donor liver-as a transplant remains the only long-term treatment available for end-stage liver failure. It is currently impossible to save everyone who needs a transplant; in Germany, every third person on the wait list for an organ dies while waiting. Part of public health care, transplant medicine in Germany is covered by the national statutory health insurance and is exclusively practised in university hospitals with specialized transplant clinics. Commissioned by the government, such national institutions as the German Medical Association (Bundesärztekammer) are crucial in defining criteria and formulating regulations regarding how organ donation and transplantation are to be practised at the national level. While the task of establishing criteria for allocating organs may seem merely a bureaucratic matter, it is these criteria that decide who in Germany should be eligible for a transplant and who should not-and thus are decisive for patients' treatment and chance of survival.

At the same time, transplant clinics and their organization, specialization and means differ significantly across the country, giving rise to a medical field shaped by particular local practices. While being a nationally regulated and locally shaped practice, German transplant medicine is also part of transnational flows of expertise and donor organs, especially as Germany is part of Eurotransplant-the non-profit organization responsible for the allocation of deceased donor organs in 8 European countries and across these countries' boundaries. With multiple organizations and institutions involved in shaping how organs in Germany are donated as well as transplanted, Arthur's desire for a liver transplant drew him into a complex web of legal, political and medical infrastructures and bureaucracies.

Bureaucracy is so ubiquitous in our lives that, as David Graeber stated, it 'has become the water in which we swim' $(2015,4)$. Dreaded by most, bureaucracies affect people differently, and while boredom or annoyance 
might ostensibly seem to be the more obvious associations with bureaucratic institutions and procedures, Monique Nuijten (2003) discusses their hope-generating characteristics. In her work on state power and its relationships with local communities in Mexico, Nuijten examines how state bureaucracies create expectations by fuelling hopes that 'everything is possible' (ibid., 16) and that 'things will be different from now on', while many of these promises remain unfulfilled. I see the features of fuelling hopes, and leaving promises unfulfilled, as constitutive elements in transplant medicine (Crowley-Matoka 2016; Kaufman and Fjord 2011; Sharp 2014) as well. With its promise to save and prolong lives, transplant medicine fuels the hopes of patients like Arthur that treatment, improvement or survival are possible. While these promises often turn out 'to be elusive' (Crowley-Matoka 2016, 150), it is the desire for more time and a better quality of life that drives people to commit to the uncertainties of this medical procedure as well as the uncertain waiting time for a transplant.

Transplant medicine in Germany relies on deceased donor organs as the main resource for liver transplants. As the death of potential organ donors is as unknown as it is unplannable and the suitability of their organs is equally uncertain, patients like Arthur wait for an indeterminate time for their hoped-for donor organ, never sure whether they will live long enough that a potential 'match' becomes available. Hope and waiting are closely related, and, as recent anthropological explorations have demonstrated, both phenomena are defined by a sense of uncertainty (Auyero 2012; Bandak and Janeja 2018; Ehn and Löfgren 2010; Elliot 2016; Hage 2009; Reed 2011). In their book on different ways of 'doing nothing' like daydreaming, routines and waiting, Billy Ehn and Orvar Löfgren $(2010)$ point to the closely connected characteristics of hope, uncertainty, liminality and waiting. 'Above all, it is the liminality of waiting that makes it a special kind of doing nothing. In-between events can make people feel stuck, but such events can also generate new possibilities' (ibid., 67). In the context of liver transplants, the new possibility people wait for is nothing less than surviving their life-threatening disease and prolonging their lives. Nonetheless, due to the many uncertainties involved and the particular requirements people on the waiting list have to meet, patients on the wait list often feel stuck too, trapped 
in a limbo, not knowing how to live between pending death or potential survival.

Ghassan Hage (2009) has explored 'stuckedness' and 'immobility' in detail by emphasizing the powerful normative discourse about people's ability to endure and 'wait out' an undesired situation. Being able to 'properly' or 'best' wait out is also of critical importance to patients waiting-in-uncertainty for a donor liver-normative discourses that become most audible and visible in transplant clinics and at patient support group meetings. Hospital and bureaucratic spaces are saturated with power relations that shape people's waiting time, issues that Sophie Day (2016) and Javier Auyero (2012) have analysed in their respective work on bureaucratic waiting for healthcare or welfare benefits. Both authors draw attention to the arbitrariness and indeterminacy of people's wait when they need either medical care (Day 2016) or welfare benefits (Auyero 2012).

The 'hope-generating machine' (Nuijten 2003) of liver transplant medicine offers patients like Arthur, who would otherwise die of organ failure, a chance of survival, fuelling the hope that they may live for a couple more years or even decades. This hope unfolds in relation to an uncertain outcome in future. Receiving a transplant remains a mere possibility throughout the patients' wait, with no guarantee that the hope for treatment will be fulfilled. Because liver transplantation is a form of life-saving treatment, the uncertainty around whether it can be actualized in time prevails throughout waiting. In other words, death is always a possibility while hopefully waiting for a liver transplant. I contend that when livers fail, the resulting existential urgencies and intimate uncertainties (Strasser and Piart 2018) shape the particularities of this form of 'expectant waiting' (Elliot 2016).

In her analysis of waiting for migration in North Africa, Alice Elliot discusses 'expectant waiting' and draws attention to the 'distinctive juxtaposition of certainty and indeterminacy' (ibid., 110) in spouses' waiting to follow their migrated partners. This expectant waiting is characterized by the waiting spouses' sense of certainty about their migration to come, while it nonetheless remains unclear when this migration will be actualized, mirroring patients' experiences of waiting for a liver transplant. Migration, or in the case of my research, a donor liver, turns into 
a constant possibility that shapes people's everyday lives, relations and subjectivities, causing 'expectant waiting' over an indeterminate period of time. While waiting for migration and for a transplant constitutes very different forms of waiting, Elliot's notion of 'expectant waiting' draws attention to the ways in which hope and expectations shape waiting experiences and how a temporal orientation to the future affects how people live in the present.

In the context of waiting for a transplant, the outcome that people hope for is to survive their life-threatening liver failure by receiving a donor organ. A crucial step towards fulfilling this hope is getting access to the waiting list, because only those on the list are considered eligible for this high-tech and high-end medical treatment. Thus, carrying the opportunities for life-saving treatment and the hope of survival, the waiting list serves as a pivotal marker of eligibility for a liver transplantunfolding in a space where the hopeful promises of transplant medicine meet healthcare infrastructures, legal frameworks and questions of triage.

\section{Lists in Flux}

Due to its centrality in allocating potentially life-saving donor livers, the waiting list requires closer consideration when thinking about technologies of waiting. Waiting lists are a central feature in German transplant medicine. They are omnipresent in patients' and physicians' narrations; they are central to medico-legal frameworks; they are mentioned in newspaper articles about the decreasing numbers of organ donations; and they played a critical role in the German transplant scandal. ${ }^{2}$ There are multiple lists and lists that overlap. Each of the 50 transplant centres in Germany manages its own waiting list, while these individual lists are also combined in an international data pool administered by

\footnotetext{
2The so-called German transplant scandal refers to physicians' wrongful tampering with patients' medical files to increase their chances of a transplant (Connolly 2013; Shaw 2013). By doing so, physicians manipulated the waiting list and interfered with patients' chance of survival. A wide-ranging matter across several clinics and transplant programmes, the scandal has led to changes in the legal regulations and structural requirements of transplant programmes in Germany.
} 
Eurotransplant in Leiden, the Netherlands. Only people registered in Eurotransplant's data pool are considered eligible for transplantation, and it is only among them that the scarce but vital resource of donor livers is allocated.

The allocation of donor livers does not operate on a 'first come, first served' principle but is based on a complex algorithm that generates 'match lists' for each donated organ that becomes available. To get into this data pool, or to 'get listed', is a crucial step towards receiving a transplant-without it, receiving a transplant in a German clinic is legally impossible. Although the notion of 'getting on the list' concerns first and foremost the inclusion of patients' data in Eurotransplant's data pool, no patient or doctor I encountered ever talked about data pools and algorithms. Patients and medical professionals talked about the waiting list, mostly how to get on the list and how to stay on it. Exactly how this list worked was of no interest to those facing a life-threatening liver disease; what they cared about was getting on the list. 'Getting on the list' was communicated by medics as the defining step on the patients' way to a transplant. While it did not mean that patients would get a transplant, getting on the list gave them a chance of a transplant.

Thus, the moment Arthur Berger had passed all necessary medical examinations and tests for a liver transplant and was considered eligible for this form of treatment, his data was forwarded to Eurotransplant and added to the international data pool administered by the non-profit organization. From that day onwards, whenever a donor liver in the Eurotransplant region became available, his data was assessed alongside that of numerous other patients' in that region to find the 'perfect match' for the available donor liver. At the same time, Arthur's clinic administered their own waiting list, of which he was part. The main reason for such local lists is that clinics are sometimes 'offered' a donor organ directly and have the opportunity to choose a suitable 'match' from among those on their own wait list. This is the case when an organ is either repeatedly declined by patients, transplant surgeons consider it unsuitable for their patients, or the organ does not meet the highest criteria and is designated as a so-called marginal organ. Patients like Arthur, whose chance of a match is limited by their age and progressive 
cancer are often asked whether they would also be willing to accept a 'marginal organ' as it significantly increases their chance for a transplant.

To make the process even more complex, the allocation of donor livers is not only based on medical criteria, but also based on ethical principles (who has the best chance of survival vs. who needs a liver most urgently), patients' body dimensions (a liver of a certain size will not fit in just any body or abdomen, but donor and recipient have to be of similar size) as well as spatial and temporal distances between clinics (to allow a quick transfer of the donor liver to its recipient). To keep the organ as healthy as possible for transplantation, this process takes place under immense time pressure, necessitating quick decisions and immediate responses. Furthermore, because of the inevitable multiple back-and-forth telephone calls between countries, clinics, medical professionals and waiting patients, those on the waiting list for a transplant have to be reachable day and night $24 / 7$. Thus, telephones, and particularly mobile phones, become critical technologies for the unfolding of transplant trajectories, particularly so for patients on the waiting list.

Unlike the digital infrastructures that these lists are now part of, lists per se are nothing new: they are among the oldest written human documents (Goody 1977, 74-111). In his investigation of literacy, Jack Goody considers lists predominantly as a tool of cataloguing, encompassing such household inventories as cattle, as well as lists of deities, kin and servants. In a general sense, generating a list creates order and reduces complexities, and sometimes the created order is hierarchical, prioritizing some things over others. Lists are a 'mode of classifying' (Goody 1977, 103) and thus intrinsically linked to processes of inclusion and exclusion. Lists make decisions visible about whom or what should or should not be put on the list. The reasoning behind what gets on a list and what does not is not always entirely clear; nevertheless, lists strive to produce the illusion that everything can be easily classified. As waiting lists for transplants are also the result of classifying practices, giving some the chance of treatment and excluding others based on medico-legal regulations, they furthermore have to be understood as a triaging tool.

These cataloguing lists that Goody is writing about are defined by their materiality and visibility and the boundaries that come with their material features. They have clear beginnings and ends; they can be read 'both 
sideways and downwards, up and down, as well as left and right' (Goody $1977,81)$. Waiting lists for transplants lack this visibility and materiality, although they present these features as snapshots in the form of temporary match lists. But these lists remain fleeting and in constant flux. Moreover, visibility is difficult to define in the context of transplant medicine with its short-lived match lists and digital data pools. Patients listed for a transplant are indeed visible to the allocating system as well as to the people administering these data sets and temporary lists. But it is only the bureaucratic, administrative aspect of this high-tech medical treatment that has an overview of all those 'on the list' and waiting for an organ. What becomes visible to the public are a wide range of numbers as well as medical conditions published in the annual reports of Eurotransplant, the German Association for Organ Donation (DSO) and local clinics. However, those who are actually waiting do not know who is waiting along with them. ${ }^{3}$

Waiting lists for a liver transplant are not worked through from the top-down, by crossing off one name after another. A clearly defined list with an obvious order does not exist in this field. What do exist are digital data sets and organ-specific algorithms of allocation that create temporary waiting lists (Amelang 2014, 21). While there is a pool of patients waiting for this form of treatment, concrete 'match lists' are formatted only if an organ becomes available and just for that one organ. The next donor liver most probably prompts the generation of a very different list. Patients' imaginings of 'getting on' the waiting list differ from the reality of accessing liver transplants by means of being added to an international data pool. Patients as well as physicians describe this stage as 'getting on the list', despite there being no actual list-at least in Goody's (1977) terms. Thus, the issue of clear readability and ordering that Goody (1977) discusses appears to be far from the waiting lists in liver transplant medicine, whose formation remains opaque for those whose name might appear under very particular circumstances on such a list.

\footnotetext{
${ }^{3}$ This is the case unless patients on the waiting list get in touch with patient associations or local support groups, which offer a platform for information and emotional support. Nonetheless, there tend to be very few patients who are waiting for a transplant at these meetings, as attendees are usually people who already received a transplant.
} 
In a strict sense, waiting lists are the result of an algorithm crossreferencing a particular donor organ with all the relevant information available about patients in Eurotransplant's data pool. When a donor organ becomes available, algorithms generate a match list and the organ is placed with a recipient in accordance with that organ-specific match list of potential organ recipients. Thus, while patients tend to think that the transplant system works to find an organ for them, it is rather that the allocating system looks for the best possible match for each organ that becomes available. Forming anew with every organ that becomes available, these 'match lists' do not hold their shape for long. Unlike a clearly defined document listing names, they are fleeting and in constant flux.

\section{Allocating Algorithms}

Due to its centrality in creating 'match lists' and thus deciding the fate of patients like Arthur, I want to take a closer look at the algorithm that is so decisive in the allocation of donor livers as well as the numbers and scores it generates. With the aim of making the allocation as fair and impartial as possible, the decision of which donor liver should be offered to whom is delegated to an algorithm that cross-references such varied data as blood type, age, height and body weight of donor and recipient to a make a match. 'Making a match is ultimately no more than pressing a button', the voice-over explains in Eurotransplant's (2015) company film, with 'advanced computer systems' behind that 'button'. After all, who or what else could make the allocation of livers-and triaging of lives_-fairer and more impartial then algorithms calculating numbers?

Highlighting the moral and political features of classifications, Bowker and Star argue that classifying schemes are 'ordinarily invisible' (2000, 2) and so ubiquitous in our everyday lives that they tend to get overlooked and taken for granted. Moreover, they point out how an increasing amount of data is organized in such highly technological and complex ways that it has become ever more challenging to trace how classifications and categories are defined, configured and employed. This is relevant to the system of transplant medicine as well, as it is a field that merges medical, political and technological terrains (Kierans and Cooper 2011). 
This invisibility becomes especially problematic when the complexities of 'decision making are hidden away inside a piece of technology or in a complex representation' (Bowker and Star 2000, 135) - like allocation algorithms.

As medical anthropologist Katrin Amelang (2014, 23-24) has pointed out, algorithms in this context represent a political compromise rather than a purely objective tool for allocation. Decisions about what kind of factors should be considered relevant in this algorithm are the product of negotiations and discussion. This is particularly so in the context of a non-profit organization that allocates organs nationally and internationally and whose member states contribute to the pool of available donor organs very differently in terms of numbers. Germany, for example, receives more donor organs from the shared pool than it contributes-in contrast to other Eurotransplant member states. ${ }^{4}$

Numbers are decisive in this field, and they are 'trusted' (Porter 1996); they decide whether a patient is too old or sick to be considered a potential transplant recipient or organ donor. They determine whether a liver is too fat, too sick, tumours too big or too numerous. Numbers form the basis on which to establish whether someone meets crucial criteria and gets included in the pool of those waiting for transplantation or not. Among all these numbers, the pivotal one for a liver transplant, though, has been the MELD (Model of End-Stage Liver Disease) score since its adoption from the United States in 2006. This score, a number between six and forty that almost every patient I met knew by heart, functions as the crucial number in liver transplant medicine. It serves as a signpost for one's chances of a transplant, as the higher the number the better one's chances. This score has real-life consequences for patients like Arthur whose data is being calculated. After being registered in Eurotransplant's

\footnotetext{
${ }^{4}$ This variation in relative numbers can be explained due to the different legal frameworks for organ donation in these countries. Unlike other member states, Germany follows an opt-in system, requiring people's active consent for organ donations. As numbers have remained low despite major campaigns promoting organ donation, a change to an opt-out system is currently being promoted by the German health minister and is causing heated debates. As transplant medicine relies on the use of human body parts and tissues, the question of organ donation remains a contested and ethically charged issue. Especially so in a country that has a history of bodies being misused for experiments under National Socialism, or bodies being considered state property in the East German socialist state (Hogle 1999, 3).
} 
data pool, and thus 'listed' for a transplant, patients' engagement with the ways in which their livers, and lives, are assessed, evaluated and classified becomes more refined and their focus on 'getting on the waiting list' is replaced by concerns about their MELD score. What patients rarely talked about was the fact that the score actually assessed their mortality risk, calculating the probability of death without a liver transplant over the next 3 months.

The so-called lab MELD score is the result of a calculation that takes three laboratory variables into account, combining serum creatinine, serum bilirubin and the international normalized ratio for prothrombin time (INR) to calculate people's mortality risk. 'The original mathematical formula for MELD is: MELD $=9.57 \times \operatorname{loge}($ creatinine $)+3.78 \times$ Loge (total bilirubin $)+11.2 \times$ Loge $($ INR $)+6.43^{\prime}$ (Kamath and Kim 2007, 797). But not all end-stage liver diseases are considered with these clearly defined laboratory values. So, for instance, in the case of liver cancer patients like Arthur, the responsible transplant committee ascribes a patient a MELD that is supposed to 'match' their mortality risk as long as their cancer stays within clearly defined criteria regarding number and size of tumours, a so-called matchMELD (Bundesärztekammer 2017). The matchMELD is intended to make the comparison between patients possible in order to assess who is most urgently in need of a transplantdespite the wide range of ways in which liver diseases present themselves, as well as the singularity of each patient's experience. These calculations are driven by the effort to assess mortality risk and control probable futures, to make the unknown predictable and manageable (Rose 2001).

Like waiting lists that are in constant flux, the MELD score is also a temporary snapshot, a number that increases and decreases with patients' fluctuating physical conditions and changing medical values. A patient's MELD score, their calculated mortality risk, not only increases with their deteriorating health, also has the potential to drop when, due to the liver's capacity to regenerate healthy tissue, a person's physical condition improves. Because the score is grounded in medical values assessed in laboratories by scientifically proven biomedical methods, it represents the dominant ideal of evidence-based medicine and is surrounded by a sense of objectivity and impartiality. Information infrastructures and technologies like scores, lists and algorithms clarify such aspects as 
patients' health, mortality risk or chance of a transplant. But while they create the impression that the decision-making processes involved are conducted fairly and objectively, these medical information technologies obscure how these are shaped by political and moral economies, intimate considerations and individual decisions (Amelang 2014; Rehsmann 2018).

The MELD score is in line with the dominant trend of the increasing standardization of medical care. Most physicians I encountered in transplant clinics would have favoured a more individualized approach to medical care and criticized this trend as well as the emphasis on evidencebased medicine. Simultaneously, many of them also felt ambivalent about more individualized medical practice. Bureaucratic paperwork was already a big part of their daily work, and while standardized forms like checklists do have their limitations (Kocman et al. 2018), they also reduce the amount of documentation required from medics. Decisions about medical treatment derived from a less standardized approach would require much more detailed documentation of all decisions involved. What is more, they would also require more time-one of the most valuable resources in healthcare institutions and a critical one when treating people with failing livers.

\section{Waiting-in-Uncertainty, Mobile Phones and Immobilities}

Arthur Berger was one of many patients I came to know in the space of the transplant clinic who was looking for treatment for their liver diseases, getting assessed for a transplant or who were already on the wait list and, thus, in the very midst of the unknowns and uncertainties of waiting for a liver transplant. Slightly older than most other patients, who were usually in their 50 s or early 60 s, the 70 -year-old was affected by irreversible liver cirrhosis as well as nonmetastatic liver cancer and without a transplant his prognosis was grim. Because no long-term substitute treatments are available for failing livers, like dialysis for kidney failure, receiving a donor liver becomes a highly urgent matter for those with irreversible liver failure. In the context of failing livers, time becomes 
an extremely limited resource-and waiting for a transplant a particularly trying experience. While Arthur was listed for a transplant, his eligibility for a donor liver was continuously reassessed by medical tests and the clinics' transplant board. For patients with liver cancer, the number as well as the size of tumours in the liver have to fit certain criteria. If their cancerous liver tumours exceed these strict limits, patients are no longer considered eligible for the treatment that they so urgently need, as they are assessed to have less chance of surviving the transplantation.

Thus, getting on the waiting list is not a unilinear process that cannot be revoked. Because of their fluctuating medical condition, patients are kept under continuous observation to assess their MELD score, to check their compliance regarding alcohol consumption or to ascertain whether their liver tumours fit the eligibility criteria. In other words, Arthur's eligibility for treatment and with it his chance of survival were not the result of a one-off assessment. Getting access to the waiting list was not the end of medical tests, treatments and assessments. What followed was a continual process of evaluation that lasted for years, with the constant threat of losing his eligibility.

In addition to the same process that all patients must go through, regardless of their age or condition, Arthur's eligibility was further scrutinized due to his advanced age-despite the fact that there is no official age limit for transplant recipients. It was his treating physician who told me that he had argued in Arthur's favour based on his biological age, because his physical constitution was that of a 60-year-old. Providing that the growth of his tumours was kept at bay, Arthur's eligibility for a liver transplant, i.e. his access to the waiting list, was granted for one more year. His doctor told me that if he did not receive a donor organ in that set timeframe, he would be considered too old and no longer eligible. So, without knowing how much time he had left without a transplant and how long he would have to wait for a donor liver, Arthur's chances got slimmer and slimmer with every month that passed by and he became increasingly impatient due to his medical emergency. His eligibility was as fragile as his health and while Arthur was glad that he was on the waiting list for a transplant, he was concerned whether he would outlive his wait. 
When I visited Arthur at home, his mobile phone was omnipresent. It was always by his side-he took it with him when he went into the kitchen, into the bathroom-and he could not bear not having it next to him. He told me that since he had got on the waiting list, he always kept his phone within reach and made sure that it was charged all the time and indeed switched on because missing a call, the call, could mean missing out on his chance of survival. As a tangible manifestation of the invisible waiting list, the phone seemed to have become his constant companion - in his daily life and in his dreams. The desire to hear his phone ring had become so strong for Arthur that he repeatedly 'heard' it ringing - mistakenly, as it turned out, again and again — and that the wished-for ringing sound even followed him into his dreams.

The mobile phone not only symbolized Arthur's hope for a transplant was also a continual reminder of his dependency on medical expertise and technologies. His imaginings of the transplant surgery followed him into the recurrent nightmares that he suffered from. Again and again, he was lying conscious and cut open on the operating table, with surgeons removing flesh and surreal objects such as a children's bicycle from his open abdomen. Again and again, he woke up in a cold sweat in the middle of the night from nightmares so unsettling that he became afraid of falling asleep. While Arthur's dream about the transplant clinic calling him on top of a mountain with his chance of a transplant was significantly less violent than these surgical nightmares, it also exemplified his unease with his life depending on a donor liver, and the sense of 'existential immobility' and 'stuckedness' he experienced while 'waiting out' (Hage 2009) his wait. With his survival being dependent on a transplant and his lack of control over the duration and outcome of his wait, Arthur felt like he had no choice but to endure this wait. Unable to live life the way he wanted, he felt stuck and suffered from the uncertainties this waiting period entailed. Nonetheless, he kept on waiting, as 'the more one waits and invests in waiting, the more reluctant one is to stop waiting' (ibid., 104), and Arthur did not want to miss out on his chance of a liver.

Glancing again and again at the phone, lying silent on the table, Arthur told me that he was bothered by the fact that he could not travel at the moment. After all, more travelling was one of the main reasons 
why he wanted a transplant. 'I'm not done yet', he said, 'I want to see so much more of the world, have more time with my wife and see my grandchildren grow up'. Arthur's mobile phone not only symbolized the possibility of treatment and the hope of prolonging his life, but was also an expression of his current immobility. If he planned to travel, he had to inform his transplant clinics, and he would temporarily be considered 'nontransplantable' for the time of his travel. He was still free to travel, but what if a donated organ became available during exactly that time? 'I couldn't bear to potentially miss out on that', Arthur said. Thus, he preferred to stay close to the clinic; 'day trips are fine, but all other trips have to wait for now'. Somehow, ironically, the mobile phone, which Arthur carried everywhere he went, seemed to make him immobile, as he had to stay close to his transplant clinic. Not being able to move as he wanted to made him feel 'like a chained yard dog with a collar around my neck', restricted in his movements and his freedom.

I want to argue that the particular workings of the 'hope-generating machine' (Nuijten 2003) of transplant medicine, with its intangible, fleeting waiting lists and its invisibly calculating algorithms, give rise to a waiting that is characterized not only by uncertainties but also by ambivalences of mobility and immobility. The mobile phone is in itself a symbol of mobility. But in the context of waiting for a liver transplant, the mobile phone symbolizes several other things, as the philosopher Francisco J. Varela (2001) pointed out in a phenomenological reflection on his liver transplantation. The procedures of being assessed and evaluated for transplantation were only the beginning of a time in which the mobile phone would become ever present:

After months I was requested to carry on me at all times a dedicated portable phone, and to never be far from the hospital. [...] Weeks without end; every minute the pressure of my portable phone as witness awakening me to the immense fragility of my life and the tenousness [sic] of my identity in this tangle of deferred causalities. (Varela 2001, 267)

The mental strain involved in being reachable day and night was also articulated by other patients, such as Erika Schneider, a woman in her 60 s who was waiting for a donor liver around the same time as Arthur: 
'This waiting definitely puts you in a state of restlessness. Because you always have to be reachable by telephone-even at night'. Even after receiving a transplant, the emotional stress of being 'always ready' for 'the call' remains a defining experience in patients' accounts. Florian Weiss, a 24-year-old who had received a liver about a year before we met, recalled his time of waiting for his transplant: 'You've got to be ready anytime; have your bag packed and the phone always in reach'. A psychologist I interviewed compared this state of restlessness and of always being-ready with the last 4 weeks of pregnancy, when expectant parents have to be ready anytime with their bags packed for the clinic. However, in the context of liver transplants this time is not limited to 4 weeks but can last indefinitely for weeks, months or even years.

It becomes clear that in the context of waiting for a liver transplant, the mobile phone is more than just a technological communication tool that makes it easier for clinics to reach patients. While the mobile phone signifies the ever-present hope for and chances of a transplant, it is also an uncomfortable reminder of one's 'fragility' (Varela 2001, 267) and 'stuckedness' (Hage 2009) in waiting for a liver. Moreover, the 'request' (Varela 2001, 267) to be reachable is often experienced as a duty that causes an additional emotional burden during waiting. Thus, while carrying the hope for a future, the mobile phone also puts a strain on the relation between the patient who is waiting and the phone that might deliver the call. Hence, I argue that the mobile phone shifts back and forth between being a mere technological communication tool and a symbol of future aspirations, medical contingencies and existential $\mathrm{im} /$ mobilities of those who are waiting for a liver.

While landlines are clearly defined in their radius of reachability, mobile phones offer the possibility of being accessible everywhere at any time, allowing increased flexibility and mobility for those waiting for their transplant clinic to call. To receive the phone call from the transplant clinic carries the potential to offer a hoped-for ending to people's waiting time and 'existential and physical immobility' (Hage 2009). The mobile phone is a reminder that the sense of 'stuckedness' that is experienced during this 'expectant waiting' (Elliot 2016) for a donor liver might lift at any time, allowing life to move on. 


\section{Conclusion}

In this chapter, I have examined the workings of transplant medicine as a 'hope-generating machine' (Nuijten 2003) by scrutinizing such critical features as waiting lists, allocating algorithms and mobile phones. I have discussed the waiting list as a crucial step for patients towards fulfilling their hope for life-saving treatment and delineated its differences from more common understandings of lists as fixed order. While lists seem to be a reasonable administrative tool to distribute goods among people, I have argued that waiting lists for liver transplants possess particularities that are better understood as articulation of transplant medicine's invisible digital infrastructure, especially due to the importance of intangible algorithms in creating these fleeting lists. By taking a closer look at the particularities of the exemplary waiting experience of Arthur Berger, I have illustrated how the invisible workings of the hope-generating transplant machinery affect those whose livers and lives are continuously assessed and evaluated while waiting for a liver. During this uncertain waiting time, patients orient their hopes according to these fleeting lists and the invisible calculations informing them. The fluctuating figure of the MELD score, which represents one's mortality risk and chance of a transplant, gives patients some indication of their hope for a donor liver and becomes a fluid benchmark symbolizing their chances of death and survival.

Liver transplant medicine is a field in which vital resources are too scarce to treat every person in need, making the death of some inevitable. Triaging livers and lives is an essential aspect of the field, in which the allocation of donor livers is delegated to waiting lists that are based on computer algorithms. With the invisible workings of these algorithms, patients' chances of survival are calculated as part of a complex digital infrastructure that generates temporary waiting lists for individual donor organs. Due to their complexity and fleeting characteristics, the powerful workings of this invisible infrastructure are hard to grasp for those waiting for a transplant and affected by it. It comes as no surprise that, instead, people talked about the waiting list rather than about multiple fleeting lists, algorithms, data pools and computer programs. 
Continuous assessment and evaluation of livers and lives by means of medical tests controls access to this temporary data pool. Hence, while waiting lists are an allocation tool, a bureaucratic technology of waiting and a legally necessary step on the way to a transplant, they also serve as a marker of eligibility and symbolize one's chances of a liver transplant. By carrying the possibility of a life-saving liver transplant, the waiting list fuels patients' hope for a future, offering a canvas for their hopes, fears and expectations. Arthur's experience illustrates how the hope for survival, and for existential and physical mobility, informs patients' decision to wait for a transplant, while this waiting then causes a sense of 'stuckedness' and generates physical and existential 'immobilities' (Hage 2009). It is the aspiration for mobility and a future that maintains patients' hope for a transplant and leads to 'expectant waiting' (Elliot 2016) that is characterized by uncertainties regarding its outcome and indeterminacy regarding its duration. I have argued that during this particular temporal experience of waiting, the mobile phone becomes an essential, but ambivalent part of the infrastructure of the 'hope-generating machine' of transplant medicine. During patients' wait for a transplant, the mobile phone, as a symbol of mobility, transforms from a mere communication tool to an ambivalent marker of people's simultaneous mobility and immobility. In this time of uncertain waiting, the mobile phone becomes an extension and tangible manifestation of the fleeting and invisible waiting list, a torturous reminder of one's fragility and dependency on medical expertise, while simultaneously always carrying the potential to mark the end to this uncertain wait.

Acknowledgements The research was funded by the Swiss National Science Foundation (project number 149368 and 175223). I would like to thank the "Intimate Uncertainties" research team, Sabine Strasser, Veronika Siegl, Gerhild Perl and Luisa Piart, as well as Janina Kehr who commented on previous versions of this chapter. Special thanks to all who shared their valuable time and experiences while waiting and hoping for a liver. 


\section{References}

Amelang, Katrin. 2014. Transplantierte Alltage: Zur Produktion von Normalität nach einer Organtransplantation. Bielefeld: Transcript Verlag.

Auyero, Javier. 2012. Patients of the State: The Politics of Waiting in Argentina. Durham and London: Duke University Press.

Bandak, Andreas, and Manpreet K. Janeja. 2018. "Introduction: Worth the Wait." In Ethnographies of Waiting: Doubt, Hope and Uncertainty, edited by M. K. Janeja and A. Bandak, pp. 1-39. London, Oxford, and New York: Bloomsbury Academic.

Bowker, Geoffrey C., and Susan Leigh Star. 2000. Sorting Things Out: Classification and Its Consequences. Cambridge, MA and London, UK: MIT Press.

Bundesärztekammer. 2017. Richtlinien zur Organtransplantation gem. $\$ 16$ TPG: Richtlinie gemäß $₫ 16$ Abs. 1 S. 1 Nrn. 2 u. 5 TPG für die Wartelistenführung und Organvermittlung zur Lebertransplantation. In Deutsches Ärzteblatt. Bundesärztekammer, ed. pp. 1-20, Vol. 114.

Connolly, Kate. 2013. "Mass Donor Organ Fraud Shakes Germany." The Guardian, January 9. Accessed May 18. https://www.theguardian.com/ world/2013/jan/09/mass-donor-organ-fraud-germany.

Crowley-Matoka, Megan. 2016. Domesticating Organ Transplant: Familial Sacrifice and National Aspiration in Mexico. Durham, NC and London: Duke University Press.

Day, Sophie. 2016. "Waiting and the Architecture of Care." In Living and Dying in the Contemporary World: A Compendium, edited by V. Das and C. Han, pp. 67-184. Oakland: University of California Press.

Ehn, Billy, and Orvar Löfgren. 2010. The Secret World of Doing Nothing. Berkeley, Los Angeles, and London: University of California Press.

Elliot, Alice. 2016. "Paused Subjects: Waiting for Migration in North Africa." Time \& Society 25 (1): 102-116.

Eurotransplant. 2015. Eurotransplant_More Than a Match. Accessed April 19, 2019. https://vimeo.com/124284017.

Goody, Jack. 1977. The Domestication of the Savage Mind. Cambridge, London, New York, and Melbourne: Cambridge University Press.

Graeber, David. 2015. The Utopia of Rules: On Technology, Stupidity, and the Secret Joys of Bureaucracy. Brooklyn and London: Melville House Publishing. 
Hage, Ghassan. 2009. "Waiting Out the Crisis: On Stuckedness and Governmentality." In Waiting, edited by G. Hage, pp. 97-106. Carlton, VIC: Melbourne University Press.

Hogle, Linda F. 1999. Recovering the Nation's Body: Cultural Memory, Medicine, and the Politics of Redemption. New Brunswick, NJ and London: Rutgers University Press.

Kamath, Patrick. S., and W. Ray Kim. 2007. The Model for End-Stage Liver Disease (MELD). Hepatology 45 (3): 797-805.

Kaufman, Sharon R., and Lakshmi Fjord. 2011. "Medicare, Ethics, and Reflexive Longevity: Governing Time and Treatment in an Aging Society." Medical Anthropology Quarterly 25 (2): 209-231.

Kierans, Ciara, and Jessie Cooper. 2011. "Organ Donation, Genetics, Race and Culture: The Making of a Medical Problem." Anthropology Today 27 (6): 11-14.

Kocman, David, et al. 2018. "Neither Magic Bullet Nor a Mere Tool: Negotiating Multiple Logics of the Checklist in Healthcare Quality Improvement." Sociology of Health \& Illness 47 (4): 1-17.

Nuijten, Monique. 2003. Power, Community and the State: The Political Anthropology of Organisation in Mexico. London and Sterling, VA: Pluto Press.

Porter, Theodore M. 1996. Trust in Numbers: The Pursuit of Objectivity in Science and Public Life. Princeton, NJ: Princeton University Press.

Reed, Adam. 2011. "Hope on Remand." Journal of the Royal Anthropological Institute 17 (3): 527-544.

Rehsmann, Julia. 2018. "Confined Live(r)s: Self-Infliction and Arbitrary Survival in the German Transplant System." Anthropological Journal of European Cultures 27 (2): 45-64.

Rose, Nikolas. 2001. "The Politics of Life Itself." Theory, Culture \& Society 18 (6): $1-30$.

Sharp, Lesley A. 2014. The Transplant Imaginary: Mechanical Hearts, Animal Parts, and Moral Thinking in Highly Experimental Science. Berkeley, Los Angeles, and London: University of California Press.

Shaw, David. 2013. "Lessons from the German Organ Donation Scandal." Journal of the Intensive Care Society 14 (3): 200-201.

Strasser, Sabine, and Luisa Piart. 2018. "Intimate Uncertainties: Ethnographic Explorations of Moral Economies across Europe." Anthropological Journal of European Cultures 27 (2): v-xv. 
Varela, Francisco J. 2001. "Intimate Distances: Fragments for a Phenomenology of Organ Transplantation." Journal of Consciousness Studies 8 (5-7): 259-271.

Open Access This chapter is licensed under the terms of the Creative Commons Attribution 4.0 International License (http://creativecommons.org/ licenses/by/4.0/), which permits use, sharing, adaptation, distribution and reproduction in any medium or format, as long as you give appropriate credit to the original author(s) and the source, provide a link to the Creative Commons license and indicate if changes were made.

The images or other third party material in this chapter are included in the chapter's Creative Commons license, unless indicated otherwise in a credit line to the material. If material is not included in the chapter's Creative Commons license and your intended use is not permitted by statutory regulation or exceeds the permitted use, you will need to obtain permission directly from the copyright holder.

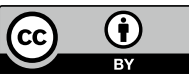

